\title{
Der lange Weg zum Perspektivenwechsel
}

\author{
Im "Aktionsplan Verbraucherschutz" der Bundesregierung erhält der Gesund- \\ heitsschutz hohe Priorität. Zwei generelle Aspekte werden im Aktionspro- \\ gramm hervorgehoben: die Orientierung am Vorsorgeprinzip sowie die Stärkung \\ von Eigenverantwortung und Selbstbestimmung der Verbraucher. Gesundheits- \\ förderung und Prävention sollen im Bereich Ernährung verbunden werden. \\ Kündigt sich damit ein sozial-ökologischer Perspektivenwechsel an?
}

G Von Ulla Simshäuser Gesundheitlicher Verbraucherschutz hatte in der Vergangenheit im wesentlichen zwei Ziele: der Staat soll die Bürger vor möglichen gesundheitlichen Risiken von Produkten, Dienstleistungen und Techniken schützen sowie über mögliche Risiken informieren. Dabei wurde implizit davon ausgegangen, dass Risiken vor allem durch bestimmte Stoffe verursacht werden und Verbraucherinformation den Bürger zum „mündigen“, sich selbst vor Gefahren schützenden Konsumenten macht.

Nun ist es längst erwiesen, dass die häufigsten Krankheiten in Deutschland wie in Europa durch den Lebensstil verursacht werden, der zwar ein bestimmtes Konsumverhalten einschließt, aber nicht darauf reduzierbar ist (1). Zu nennen sind Fehlernährung (insbesondere Adipositas), Bewegungsmangel und psychische Belastungen. An der Spitze der Erkrankungen stehen Herz-KreislaufKrankheiten, gefolgt von Karies, Krebs und Diabetes. Zum einen sind Frauen und Männer von diesen Krankheiten unterschiedlich betroffen, zum anderen konzentrieren sich diese Erkrankungen auf besonders verletzliche soziale Gruppen wie Arme, Alte, Kinder- und Jugendliche sowie MigrantInnen.

Gesundheitsberatung steht aber vor dem grundsätzlichen Dilemma, dass Informationsstrategien den mündigen Bürger schaffen wollen, ihn aber gleichzeitig voraussetzen. Denn Informationsstrategien beziehen sich auf den Lebensstil einer bürgerlichen, eher männlichen Mittelschicht, die nicht nur schnellen Zugriff zu Information fordert, sondern diese auch zu bewerten und zu selektieren weiß, und die aufgrund ihrer Möglichkeiten davon ausgeht, dass sie das eigene Leben aktiv und selbstbestimmt gestalten kann (siehe auch die Beiträge von Schultz und Rehaag et al. in diesem Heft). Gerade die in gesundheitlicher Hinsicht verletzlichen Gruppen werden durch die bisher üblichen Informationsstrategien nicht erreicht.

\section{- Soziale Herausforderungen annehmen}

Die dramatische Krise der sozialen Sicherungssysteme in Folge von demografischem Wandel und globalen wirtschaftlichen Veränderungen stellt den Verbraucherschutz vor neue Aufgaben. Nur gesunde, zur Selbsthilfe fähige Bürger können eigene Sicherungssysteme aufbauen, den Staat entlasten und die Wirtschaft fördern. Voraussetzung dafür ist, dass VerbraucherInnen im Alltag gesundheitsfördernde Kompetenzen entwickeln und anwenden können. Auf den dafür erforderlichen organisatorischen und institutionellen Wandel im Sinne der Förderung von psychischem und sozialem Wohlbefinden und von Lebensqualität, so genannter Salutogenese, zielt Gesundheitsförderung. Dort, wo viele Menschen täglich arbeiten, lernen oder ihre Freizeit verbringen, werden Organisationsentwicklungsprojekte für wünschenswerte Veränderungen im Alltag mit und aus Sicht der Beteiligten durchgeführt. Auf diese Weise können mehr Menschen und insbesondere verletzliche Gruppen erreicht werden, als mit individueller Aufklärung.

Entsprechende Praxisbeispiele untersucht das IÖW derzeit für Schulen im Rahmen des Verbundvorhabens „Ernährungswende“ (2). So werden in gesundheitsfördernden Schulen Ernährungsangebote gemeinsam mit Schülern entwickelt, Pausenregelungen im Sinne der Gesundheits- und Bewegungsförderung nach ihren Vorstellungen verändert, im
Unterricht ernährungs- und gesundheitsrelevante Inhalte gelehrt sowie Aggressions- und Stressbewältigung eingeübt. Auf diese Weise sollen gesundheitsfördernde Kompetenzen, Werte für Lebensqualität entwickelt und entsprechende Alltagsroutinen aufgebaut werden.

Die Veränderung von Räumen des Alltags wie Schulen, Betriebe, Krankenhäuser und Gemeinden (auch settings für Gesundheit genannt), ist kein singulärer Ansatz sondern seit langem Kernphilosophie und Schlüsselstrategie der Weltgesundheitsorganisation (WHO) (3). Mit den zuletzt 1998 verabschiedeten Gesundheitszielen für Europa im 21. Jabrhundert verpflichten sich die WHO-Mitgliedstaaten unter anderem den ,Ersten Aktionsplan Lebensmittel und Ernährungspolitik“ der WHO in ihren Ländern umzusetzen. Er sieht Maßnahmen zur Risikoprävention und -kommunikation für eine gesunde Ernährung bei Kindern und Jugendlichen vor. In Deutschland wird er derzeit im „Aktionsprogramm Umwelt und Gesundheit (APUG)" unter anderem mit Unterstïtzung des Verbraucherministeriums (BMVEL) umgesetzt. Ein weiteres WHO- Gesundheitsziel ist die Bildung von settings für Gesundheit. Bis 2005 sollten:

- mindestens 50Prozent aller Kinder in Deutschland die Möglichkeit haben, einen gesundheitsfördernden Kindergarten zu besuchen,

- 95Prozent aller Kinder die Möglichkeit haben, eine gesundheitsfördernde Schule zu besuchen,

- sich mindestens 50Prozent aller Städte, städtischen Ballungsgebiete und Gemeinden aktiv an einem Netzwerk gesunder Städte beziehungsweise Gemeinden beteiligen,

- sich mindestens 10Prozent aller mittleren und großen Unternehmen zur Umsetzung der Prinzipien eines gesunden Unternehmens verpflichten (4).

Ob diese Ziele in Deutschland erreicht werden, dürfte eher fraglich sein, denn Deutschland beschreitet in Sachen Gesundheitsförderung den Weg einer nachholenden Entwicklung. Während sich das Öffentliche Gesundheitswesens in vielen Europäischen Ländern (zum Beispiel die Schweiz, Schweden, England) an salutogenetischen Ansätzen orientiert, tut man sich hierzulande nicht zuletzt aufgrund der Strukturen im Gesundheitswesen schwer. Obwohl in WHO Netzwerken wie Gesunde-Städte viele praktische Erfahrungen gesammelt wurden, ist es bisher nicht gelungen, diese in programmatische Politikansätze einzubinden (5).

Gesundheitlicher Verbraucherschutz kann und muss mehr sein als Risikokommunikation und 
Verbraucherinformation. Neben der dringend erforderlichen Stärkung der Patientenrechte im Gesundheitswesen muss er auch dem gesundheitsrelevanten Wandel im Alltag von Männern und Frauen Rechnung tragen (6). Nur mit einer aktiven Gesundheitsförderung bleiben VerbraucherInnen der Wirtschaft als KonsumentInnen und Arbeitnehmerinnen erhalten und garantieren staatliche Handlungsfähigkeit im Zeichen begrenzter nationalstaatlicher Steuerungsmöglichkeiten. Indem durch Gesundheitsförderung nicht mehr nur Akteure entlang von Märkten und Produktlinien sondern auch Akteure aus alltäglichen Handlungskontexten wie dem Arbeitsplatz oder der Kindertagesstätte in den Blick rücken, eröffnen sich neue Möglichkeiten für gesundheitlichen Verbraucherschutz.

\section{- Risikoprävention und Gesundheitsförderung}

Ein gesundheitlicher Verbraucherschutz, der produktbezogene und salutogenetische Ansätze integriert, hebt die bisher vorhandene Trennung zwischen Gesundheitsförderung, Sozial- und Wirtschaftspolitik auf und macht ihn zur Querschnittsaufgabe. Er rückt Demokratisierung der Marktbeziehungen in den Mittelpunkt und integriert auch Strategien für Geschlechtergerechtigkeit und die Förderung sozial benachteiligter Gruppen.

Der Aktionsplan des BMVEL eröffnet Chancen zur Entwicklung eines sozial-ökologischen Paradigmas. Obwohl die im Aktionsplan skizzierten Maß- nahmen noch stark am Modell der individuellen Risikokommunikation und -prävention orientiert sind, wird doch bereits ,die rasche Verzahnung und Verstärkung der verschiedenen Aktivitäten im Bereich von Prävention und Gesundheitsförderung“ zumindest als Ziel formuliert. Ein erster wichtiger Schritt in diese Richtung ist die im November 2002 angetretene Mitgliedschaft des BMVEL im Deutschen Forum Prävention und Gesundheitsförderung. Zu wünschen bleibt, dass die Umsetzung des Ziels nicht auf halbem Wege stecken bleibt und Erfahrungen von Gesundheitswissenschaft und Gesundheitsförderung auch in konzeptioneller Hinsicht genutzt werden.

\section{Sozial-ökologische Forschung für eine Veränderung der Praxis}

Ein Wandel im Verständnis von gesundheitsbezogenem Verbraucherschutz fordert von der Wissenschaft ein transdisziplinäres, problemorientiertes Vorgehen. Ökologische Wirtschaftsforschung bedeutet hier nach Praxisansätzen zur Verbindung der Handlungsfelder Umwelt, Gesundheit und Wirtschaft zu fragen. Aufzuzeigen wären Wege der praktischen Zusammenführung etwa von ökologischer Produktpolitik, gesundheitsbezogenem und betrieblichem Umweltschutz oder Möglichkeiten für sozial-ökologische Dienstleistungen, die Wohlbefinden und Alltagskompetenz von VerbraucherInnen, stärken und damit zu einer Entlastung von Sicherungssystemen beitragen.

\section{Anmerkungen}

(1) WHO: The European Health Report, WHO Regional Publications, European Series, №. 97, 2002

(2) Das Projekt „Ernährungswende" ist ein transdisziplinäres Forschungsvorhaben gefördert mit Mitteln des BMBF im Rahmen des Förderprogramms Sozial-ökologische Forschung. Partner im Forschungsverbund sind: Öko-Institu† (Gesamtprojektleitung), Institut für ökologische Wirtschaftsforschung, Institut für sozial-ökologische Forschung, Katalyse-Institut für angewandte Umwelfforschung.

(3) Grossmann, Ralph, Scala, Klaus: Gesundheit durch Projekte fördern, ein Konzept zur Gesundheitsförderung durch Organisationsentwicklung und Projektmanagement, Weinheim/ München, 1996

(4) WHO Regionalbüro für Europa: Das Rahmenkonzept: "Gesundheit für alle" für die Europäische Region der WHO, Kopenhagen, 1999

(5)Trojahn, Alf, Leggewie, Klaus: Nachhaltige Gesundheit und Entwicklung., in: Wechselwirkung Heft 6, 43-47, 2002

(6) Verbraucherzentrale Bundesverband (VZBV): Verbraucherpolitische Prüfsteine zur Bundestagswahl, Berlin, 2002

Die Autorin
Ulla Simshäuser ist wiss. Mitarbeiterin in den For-
schungsfeldern ökologische Produktpolitik und ökolo-
gischer Konsum des Inst. für ökologische Wirtschaftsfor-
schung (IÖW).
Kontakt: IÖW-Büro Heidelberg, Bergstraße 7,
69120 Heidelberg. Tel. 06221-649160,
E-Mail: Ulla.Simshaeuser@heidelberg.ioew.de

\section{Der GENaue Blick}

\section{Grüne Gentechnik auf dem Prüfstand}

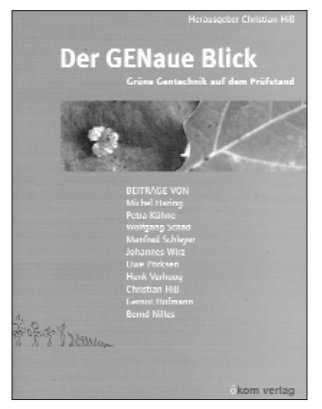

Christian Hiß, Herausgeber, ökom Verlag,

München 2002

160 Seiten,

ISBN 3-936581-01-0,

Preis 16,- $€$

(zzgl. Versandkosten)
Die Grüne Gentechnik verändert die Landwirtschaft, ihre Früchte und die Gesellschaft. Dieses Buch erhebt den Anspruch, die Gentechnik-Debatte über die Konfrontation von Risiko und Nutzen hinweg zu heben. Es bietet eine Grundlage für die Urteilsbildung des Lesers, der als moralisch handelnder Mensch und nicht nur mit dem Verstand des Ökonomen, Naturwissenschaftlers oder Juristen die Gentechnologie beurteilen will. Zehn Autoren aus unterschiedlichen Wissens- und Arbeitsgebieten stellen die Grüne Gentechnik kritisch auf den Prüfstand. Diese Bündelung "GENauer Blicke» liefert dem Leser Hintergründe, Grundlagen und Fakten.

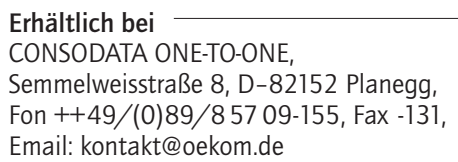


(c) 20I0 Authors; licensee IÖW and oekom verlag. This is an article distributed under the terms of the Creative Commons Attribution Non-Commercial No Derivates License (http://creativecommons.org/licenses/by-nc-nd/3.o/), which permits unrestricted use, distribution, and reproduction in any medium, provided the original work is properly cited. 